Original Research Article

\title{
A comparative study of efficacy and safety of topical calcitriol and topical calcipotriol in stable chronic plaque type psoriasis
}

\author{
Leela Hugar ${ }^{1}$, Ramesh H. ${ }^{2 *}$
}

${ }^{1}$ Department of Pharmacology, BM Patil Medical College, Vijayapura, Karnataka, India ${ }^{2}$ Department of Pharmacology, Karnataka Institute of Medical Science, Hubli, Karnataka, India

Received: 16 January 2019 Accepted: 09 February 2019

\section{*Correspondence to:}

Dr. Ramesh H.,

Email: kimsramesh@ yahoo.co.in

Copyright: (C) the author(s), publisher and licensee Medip Academy. This is an openaccess article distributed under the terms of the Creative Commons Attribution NonCommercial License, which permits unrestricted noncommercial use, distribution, and reproduction in any medium, provided the original work is properly cited.

\begin{abstract}
Background: Topical calcitriol and calcipotriol, the two vitamin D derivatives although considered efficient in treating psoriasis, their comparative studies are relatively scanty. The objective of the present study was to evaluate and compare the efficacy and safety of calcitriol and calcipotriol in stable chronic plaque-type psoriasis.

Methods: Total 50 patients of chronic stable plaque-type psoriasis were randomly divided into two groups of 25 each. One group received calcitriol $3 \mu \mathrm{g} / \mathrm{g}$ ointment and the other group received calcipotriol $50 \mu \mathrm{g} / \mathrm{g}$ ointment twice daily for 12 weeks. Efficacy evaluations comprised global improvement (on a 4-point scale from 0: no change, to 3: clear or almost clear) assessed clinically and by the subject. Efficacy further included the 'dermatological sum score' (DSS) at each study visit. Safety evaluations (on a 5-point scale from 0: none, to 4: very severe) included clinical assessment of cutaneous safety and assessment of cutaneous discomfort by the subject.

Results: Both calcitriol and calcipotriol were significantly effective ( $p<0.001)$ in reduction of DSS but the difference between the two groups was not statistically significant. Mean score of global improvement assessed clinically was 2.20 for calcitriol and 2.16 for calcipotriol $(\mathrm{p}>0.05)$ and by the subject was 1.92 for calcitriol and 1.84 for calcipotriol $(\mathrm{p}>0.05)$. The difference between the two groups was not statistically significant. The mean worst score for cutaneous safety was higher in calcipotriol group compared to calcitriol group ( 0.28 vs 0.04 and 0.36 vs 0.04 by clinically and by the subject, respectively). Statistically significant better safety profile $(\mathrm{p}<0.05)$ was seen for calcitriol, only when assessed by the subject. $24 \%$ treatment related adverse events were reported with calcipotriol against only $4 \%$ with calcitriol.

Conclusions: Topical calcitriol and calcipotriol showed similar efficacy in the treatment of chronic plaque psoriasis while calcitriol showed better safety profile in comparison with calcipotriol, in terms of local tolerance and induced less treatment related adverse events.
\end{abstract}

Keywords: Calcipotriol, Calcitriol, Cutaneous safety, Cutaneous discomfort, Global assessment, Plaque type psoriasis

\section{INTRODUCTION}

Psoriasis is a common, chronic, disfiguring, inflammatory and proliferative condition of the skin, in which both genetic and environmental influences have a critical role. ${ }^{1}$ The disease has a worldwide distribution and affects men and women of all ages. Prevalence of psoriasis varies in different parts of the globe, ranging from $0.5 \%$ to close to $2.5 \% .^{2}$ Chronic stable plaque psoriasis (Psoriasis vulgaris) is the most common form of psoriasis, seen in approximately $90 \%$ of patients. Red, scaly, symmetrically distributed plaques are characteristic. ${ }^{3}$ The pathophysiology of psoriasis is characterized by epidermal hyperproliferation, enhanced antigen presentation, helper 
T-cell Th1 and Th17 cytokine production, T-cell expansion and angiogenesis. ${ }^{4}$ It is a classical Th1 mediated disorder with TNF- $\alpha$ being the predominant cytokine. ${ }^{5}$ Recently, cytokines IL-12 and IL-23 have also been implicated in the pathogenesis of psoriasis. IL-12 promotes growth and differentiation of naïve T-cells into Th1 and cytotoxic T- cells, whereas IL-23 enables survival and proliferation of Th17 cells. ${ }^{6,7}$ When a single disease of unknown etiology has multiple alternative modes of therapy, it is obvious that no one form of therapy is ideal. This is especially true of psoriasis for which multiple alternative treatments are used. Newer aspects of therapies or therapeutic approaches are added frequently. These many forms of therapies have their own benefits and drawbacks, often no single treatment is ideal, and it is rare for a patient not to receive several alternative treatments during his or her life time.

Although no cure is available, the disease can be effectively controlled by various therapeutic options. To date a big array of topical therapies are available for the treatment of psoriasis, in particular for mild-to- moderate plaque type psoriasis. They include traditional treatments, such as dithranol, topical retinoids, salicylic acid, tar, corticosteroids, and more recently topical vitamin D

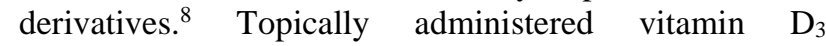
derivatives for the treatment of psoriasis are: calcipotriol, tacalcitol, maxacalcitol and more recently calcitriol. ${ }^{1,9}$ Topical formulations of calcipotriol and other vitamin $\mathrm{D}_{3}$ analogues are probably the most widely prescribed active topical therapy for plaque psoriasis. Topical calcipotriol although considered efficient, its superiority over topical calcitriol is not well established. Therefore, this study is undertaken to compare the efficacy and safety of topical calcitriol with that of calcipotriol in stable chronic plaque type psoriasis.

The objective of the present study was to evaluate and compare the efficacy and safety of calcitriol and calcipotriol in stable chronic plaque-type psoriasis.

\section{METHODS}

The design of this study was Randomized, open label, parallel, comparative study conducted at Department of Dermatology, KIMS, Hubali, Karnataka, India between October 2012 to October 2013.

\section{Sample size}

Sample size was calculated by the formula

Patients per group $=\mathrm{f}(\alpha, \beta) \times 2 \times \mathrm{SD}^{2} /(\mathrm{d})^{2}$

The minimum sample size required was 25 patients in each group. This was calculated to detect a difference of 4 in mean DSS between the groups with $80 \%$ power and 0.05 probability of type 1 error, assuming standard deviation of 5 in DSS.

\section{Inclusion criteria}

- $\quad$ Both male and female subjects of age group 18 to 70 years.

- Subjects with stable chronic plaque psoriasis.

- Body surface area involvement less than $35 \%$.

\section{Exclusion criteria}

- Patients with unstable, acute guttate, pustular, erythrodermic or orthropathic psoriasis.

- Patients with history of hypercalcaemia, renal dysfunction, calcium-based calculi, underlying conditions that require the use of systemic supplements of calcium or vitamin D.

- $\quad$ Body surface area involvement $>35 \%$.

- Subjects who had applied topical antipsoriatic medication within past 2 weeks or had used systemic antipsoriatic medication within the past 8 weeks.

- Patients who had other extensive skin disease and who had severe systemic illness.

- Pregnant women.

After obtaining clearance from Institutional ethics committee, fifty patients of chronic plaque psoriasis were recruited for the study based on inclusion and exclusion criteria. Randomisation of the subjects was done in $1: 1$ ratio, into two study groups (calcitriol and calcipotriol group) by computer generated random numbers with 25 subjects in each group.

\section{Calcitriol group}

Subjects in this group were treated with Calcitriol $3 \mu \mathrm{g} / \mathrm{g} .{ }^{10,11}$ (Lifestar pharma, Delhi) ointment applied twice daily for 12 weeks.

\section{Calcipotriol group}

Subjects in this group were treated with calcipotriol $50 \mu \mathrm{g} / \mathrm{g} .{ }^{1,9,10}$ (Biocon biopharmaceuticals, Bangalore) ointment applied twice daily for 12 weeks.

\section{Assessment of efficacy and safety}

\section{Assessment of efficacy}

- Global assessment of improvement

- Dermatological sum score (DSS)

\section{Global assessment of improvement ${ }^{10}$}

- Primary efficacy criteria- It is the global assessment of improvement done clinically by the dermatologist at the end of the study (week 12).

- Secondary efficacy criteria: It is the global assessment of improvement as assessed by the subject at the end of study (week 12). 
Global assessment of improvement was rated on a 4-point scale.

- 0 - No change

- 1 - Some improvement

- 2 - Marked improvement

- 3 - Clear or almost clear

The baseline score (week 0) was taken as zero and the total score was recorded at the end of the study (week 12).

\section{Dermatological sum score $(D S S)^{10}$}

It was the sum of erythema, plaque elevation and scaling of target lesion. Each sign was evaluated on a 5-point scale at each visit.

- 0 - None

- 1 - Mild

- 2 - Moderate

- 3 - Severe

- 4 - Very severe.

The total score was calculated at baseline (week 0) and at the end of study (week 12).

\section{Assessment of safety ${ }^{10}$}

- Clinical assessment of cutaneous safety by dermatologist

- Assessment of cutaneous discomfort by the subject.

\section{Clinical assessment of cutaneous safety by dermatologist}

Local reaction due to the given topical treatment was assessed by the dermatologist. It was done on a 5-point scale.

- $\quad 0$ - None (No local reaction)

- 1 - Mild (Localized, perceptible erythema)

- 2 - Moderate (Bright red erythema with/without edema confined to the area of application)

- 3 - Severe (Erythema with edema extending beyond the area of application)

- 4 - Very severe (Vesiculation/ erosion/ ulceration)

The baseline score (week 0) was taken as zero and the total score was recorded at the end of the study (week 12).

\section{Assessment of cutaneous discomfort by the subject}

The discomfort experienced by the subject was assessed on a 5-point scale.

- $\quad$-No discomfort

- 1-Mild discomfort

- 2-Moderate discomfort

- 3-Severe discomfort

- 4- Very severe discomfort.
The baseline score (week 0) was taken as zero and the total score was recorded at the end of the study (week 12).

\section{Follow up}

The patients were followed up at 2-weeks, 4-weeks, 8weeks and at 12-weeks of completion of treatment in both the groups. The follow up included the following.

- Assessment of DSS (sum of erythema, plaque elevation and scaling of target lesion)

- Assessment of global improvement.

- Any adverse effects due to the medication were noted and score for assessment of safety was given at 12weeks of completion of treatment.

\section{Statistical analysis}

Descriptive statistical analyses were carried out in the present study. Results on continuous measurements were presented on Mean \pm SD and results on categorical measurements are presented in Number $(\%)$.

Non-parametric test was applied for the data not have been normally distributed; Wilcoxon Signed Ranks test for intra group comparison and Mann-Whitney test for inter group comparison. For the entire test a p-value of 0.05 or less was considered for statistical significance.

\section{RESULTS}

\section{The baseline clinical variables}

Mean age, mean body surface area, percentage of males and females showed no statistically significant difference between the two groups and hence both the groups are comparable $(\mathrm{p}>0.05)$ (Table 1).

Table 1: Pre-treatment clinical variables of patients.

\begin{tabular}{|llll|}
\hline Variables & $\begin{array}{l}\text { Calcitriol } \\
\text { group } \\
(\mathbf{n = 2 5})\end{array}$ & $\begin{array}{l}\text { Calcipotriol } \\
\text { group } \\
(\mathbf{n = 2 5})\end{array}$ & $\begin{array}{l}\text { p- } \\
\text { value }\end{array}$ \\
\hline $\begin{array}{l}\text { Mean age } \\
\text { (years) } \pm \text { SD }\end{array}$ & $42 \pm 12.29$ & $44.0 \pm 12.02$ & $0.73^{*}$ \\
\hline Females (\%) & 40 & 40 & \\
\hline Males (\%) & 60 & 60 & $0.96^{*}$ \\
\hline $\begin{array}{l}\text { Mean BSA } \\
(\%) \pm S D\end{array}$ & $9.04 \pm 4.80$ & $9.12 \pm 6.26$ & \\
\hline
\end{tabular}

* Unpaired t-test; $\mathrm{p}>0.05$ - Non-significant. The baseline clinical variables were compared with unpaired t-test. There was no statistically significant difference between the two groups ( $p$ $>0.05$ ) and hence both the groups are comparable.

The mean baseline DSS of calcitriol group is $8.16 \pm 2.26$ and mean baseline DSS of calcipotriol group is $8.24 \pm 2.06$. There was no statistically significant difference between the groups ( $p>0.05)$ (Table 2). 


\section{Calcitriol group}

Mean DSS at baseline was 8.16 \pm 2.26 and mean DSS at 12 wks was $1.40 \pm 1.41$. The reduction of mean DSS at 12 weeks post treatment compared to baseline was found to be statistically highly significant $(\mathrm{P}<0.001)$ (Table 3$)$.

Table 2: Pre-treatment DSS scores in both the groups.

\begin{tabular}{|lllc|}
\hline Variables & $\begin{array}{l}\text { Calcitriol } \\
\text { group }(\mathbf{n}=25)\end{array}$ & $\begin{array}{l}\text { Calcipotriol } \\
\text { group }(\mathbf{n}=25)\end{array}$ & $\begin{array}{c}\mathbf{p}- \\
\text { value }\end{array}$ \\
\hline $\begin{array}{l}\text { Mean age } \\
\text { (years) } \pm \text { SD }\end{array}$ & $42 \pm 12.29$ & $44.0 \pm 12.02$ & $0.73^{*}$ \\
\hline
\end{tabular}

*Mann-Whitney test; p>0.05 - Non-significant. The baseline DSS between the two groups was compared with Mann- Whitney test. There was no statistically significant difference $(p>0.05)$ between the groups.

Table 3: Calcitriol group: mean DSS at 12 weeks post treatment compared to baseline.

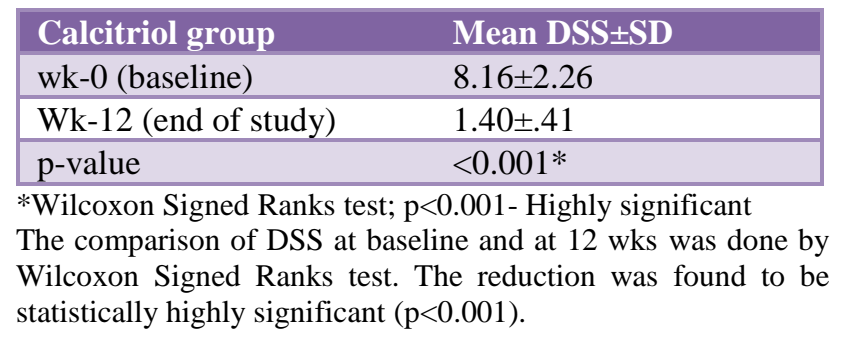

\section{Calcipotriol group}

Mean DSS at baseline was 8.24 \pm 2.06 and mean DSS at 12 wks was $1.36 \pm 1.25$. The reduction of mean DSS at 12 weeks post treatment compared to baseline was found to be statistically highly significant $(\mathrm{P}<0.001)$ (Table 4$)$.

Table 4: Calcipotriol group: mean DSS at 12 weeks post treatment compared to baseline.

\begin{tabular}{|ll|}
\hline Calcipotriol group & Mean DSS \pm SD \\
\hline wk-0 (baseline) & $8.24 \pm 2.06$ \\
\hline Wk-12 (end of study) & $1.36 \pm 1.25$ \\
\hline p-value & $<0.001^{*}$ \\
\hline
\end{tabular}

*Wilcoxon Signed Ranks test; $\mathrm{p}<0.001$ - Highly significant. The comparison of DSS at baseline and at 12 wks was done by Wilcoxon Signed Ranks test. The reduction was found to be statistically highly significant $(\mathrm{p}<0.001)$.

Table 5: Mean DSS compared between calcitriol group and calcipotriol group at wk-2, wk-4, wk-8 and wk-12.

\begin{tabular}{|llll|}
\hline Week & $\begin{array}{l}\text { Calcitriol group } \\
\text { Mean DSS } \pm \text { SD }\end{array}$ & $\begin{array}{l}\text { Calcipotriol } \\
\text { group } \\
\text { Mean DSS } \pm \text { SD }\end{array}$ & $\begin{array}{l}\text { p- } \\
\text { value }\end{array}$ \\
\hline Wk- 2 & $6.24 \pm 2.14$ & $6.40 \pm 1.77$ & 0.875 \\
\hline Wk-4 & $4.32 \pm 1.77$ & $4.48 \pm 1.44$ & 0.704 \\
\hline Wk-8 & $2.72 \pm 1.62$ & $2.84 \pm 1.40$ & 0.866 \\
\hline Wk-12 & $1.40 \pm 1.41$ & $1.36 \pm 1.25$ & 0.960 \\
\hline
\end{tabular}

Mann-Whitney test; $\mathrm{p}>0.05$ - Non-significant. On comparing mean DSS of both groups, there was no statistically significant difference between calcitriol group and calcipotriol group at 2 weeks, 4 weeks, 8 weeks and at 12 weeks of treatment.

On comparing mean DSS of both groups, there was no statistically significant difference between calcitriol group and calcipotriol group at 2 weeks, 4 weeks, 8 weeks and at 12 weeks of treatment $(\mathrm{P}>0.05)$ (Table 5).

\section{Global assessment of improvement}

The mean GA score as assessed clinically at 12 wks in calcitriol group was 2.1600 .687 and in calcipotriol group was $2.200 \pm 0.707$. The mean GA score as assessed by subject at 12 wks in calcitriol group was $1.920 \pm 0.909$ and in calcipotriol group was $1.840 \pm 0.898$. On comparing mean GA scores of both groups, there was no statistically significant difference between two groups at 12 weeks clinically as well as by the subject $(\mathrm{P}>0.05)$ (Table 6).

Table 6: Global assessment of improvement compared between calcitriol group and calcipotriol group, clinically and by the subject at wk-12.

\begin{tabular}{|llll|}
\hline Group & Particulars & $\begin{array}{l}\text { GA } \\
\text { clinically } \\
\text { Wk-12 }\end{array}$ & $\begin{array}{l}\text { GA by } \\
\text { subject } \\
\text { Wk-12 }\end{array}$ \\
\hline $\begin{array}{l}\text { Calcitriol } \\
\text { group (I) }\end{array}$ & Mean \pm SD & $2.160 \pm 0.687$ & $1.920 \pm 0.909$ \\
\hline $\begin{array}{l}\text { Calcipotriol } \\
\text { group (II) }\end{array}$ & Mean \pm SD & $2.200 \pm 0.707$ & $1.840 \pm 0.898$ \\
\hline I vs II & p-value & 0.823 & 0.726 \\
\hline
\end{tabular}

Mann-Whitney test; $\mathrm{p}>0.05$ - Non-significant.

The GA scores of calcitriol group and calcipotriol group at $12 \mathrm{wks}$ was compared by using Mann-Whitney test. There was no statistically significant difference between these group at 12 weeks (end of study) of treatment clinically as well as by the subject.

Safety assessment compared between the calcitriol group and calcipitriol group clinically and by the subject at week 12.

\section{Clinical assessment of cutaneous safety}

Table 7: Safety assessment compared between the calcitriol group and calcipotriol group, clinically and by the subject at wk-12.

\begin{tabular}{|llll|}
\hline Group & Particulars & $\begin{array}{l}\text { Clinically } \\
\text { Wk-12 }\end{array}$ & $\begin{array}{l}\text { By subject } \\
\text { Wk-12 }\end{array}$ \\
\hline $\begin{array}{l}\text { Calcitriol } \\
\text { group (I) }\end{array}$ & Mean \pm SD & $0.040 \pm 0.200$ & $0.040 \pm 0.200$ \\
\hline $\begin{array}{l}\text { Calcipotriol } \\
\text { group (II) }\end{array}$ & Mean \pm SD & $0.28 \pm 0.613$ & $0.360 \pm 0.700$ \\
\hline I vs II & p-value & $0.079 *$ & $0.039 * *$ \\
\hline
\end{tabular}

Mann-Whitney test $* \mathrm{P}>0.05$ - Non-significant. $* * \mathrm{P}<0.05-$ significant 
The mean score for cutaneous safety was higher in calcipotriol group compared to calcitriol group (0.28 vs $0.04)$. But there was no statistically significant difference between the groups $(\mathrm{P}>0.05)$ (Table 7).

\section{Assessment of cutaneous discomfort by the subject}

The mean score for cutaneous discomfort was higher in calcipotriol group compared to calcitriol group (0.36 vs 0.04). It was statistically significant $(\mathrm{P}<0.05)$ (Table 7).

\section{DISCUSSION}

\section{Pre-treatment clinical variables of patients}

The pre-treatment clinical variables of patients in both the treatment groups were compared and analysed. All variables like age of patients, sex ratio and BSA $(\%)$ in both groups are comparable. The baseline DSS scores in both groups showed no statistically significant difference. The two treatment groups were comparable with regard to pertinent clinical variables at baseline.

\section{Assessment of efficacy}

Dermatological sum score and global assessment of improvement

\section{Calcitriol group}

In the present study calcitriol $3 \mu \mathrm{g} / \mathrm{g}$ ointment was effective in the treatment of stable chronic plaque psoriasis, which was statistically highly significant $(\mathrm{P}<0.001)$ in terms of reduction in DSS.

In a study conducted by Langner A et a1, chronic plaque psoriasis treated with calcitriol in concentrations of $3 \mu \mathrm{g} / \mathrm{g}$ and $15 \mu \mathrm{g} / \mathrm{g}$ improved significantly. ${ }^{11}$ The use of higher dose $(15 \mu \mathrm{g} / \mathrm{g})$ did not show any clinical superiority over calcitriol $3 \mu \mathrm{g} / \mathrm{g}$, in contrast it was associated with a higher risk of hypercalciuria when applied to extensive skin lesions. Because of these findings, a concentration of $3 \mu \mathrm{g} / \mathrm{g}$ is considered to be optimal. The clinical effectiveness was confirmed by the histopathological evaluation after calcitriol treatment, with marked normalization of all most all histological features typical for psoriasis.

In two placebos controlled multicentric randomized double-blind parallel group studies, $3 \mu \mathrm{g} / \mathrm{g}$ calcitriol ointment was compared with its excipient. In both studies the drug was shown to be significantly effective in mild-tomoderate plaque psoriasis than its excipient. ${ }^{12}$ The present study is in concurrence with the above studies in showing the efficiency of calcitriol $3 \mu \mathrm{g} / \mathrm{g}$ ointment in the treatment of chronic plaque psoriasis.

\section{Calcipotriol group}

In the present study calcipotriol $50 \mu \mathrm{g} / \mathrm{g}$ ointment was also effective in the treatment of stable chronic plaque psoriasis, which was statistically highly significant $(\mathrm{P}<0.001)$ in terms of reduction in DSS.

Ashcroft DM in his quantitative systematic review of randomised controlled trials with 6038 patients with plaque psoriasis reported in 37 trials where patients were treated with calcipotriol ointment $50 \mu \mathrm{g} / \mathrm{g}$, concluded that calcipotriol is effective in the treatment of mild to moderate chronic plaque psoriasis. ${ }^{13}$

Scott et al, presented an overview of studies on the use of calcipotriol ointment in the management of psoriasis. ${ }^{14}$ They confirmed the efficacy of calcipotriol ointment applied twice daily and concluded that calcipotriol ointment is valuable as a first or second-line therapy option for the management of mild to moderate psoriasis.

The present study is in concurrence with the above studies in showing the efficiency of calcipotriol $50 \mu \mathrm{g} / \mathrm{g}$ ointment in the treatment of chronic plaque psoriasis.

\section{Calcitriol group vs calcipotriol group}

On comparison between treatment with calcitriol $3 \mu \mathrm{g} / \mathrm{g}$ ointment and calcipotriol $50 \mu \mathrm{g} / \mathrm{g}$ ointment at 2 weeks, 4 weeks, 8 weeks and at 12 weeks, there was no statistically significant difference $(\mathrm{p}>0.05)$ between the two groups at all post baseline time-points in terms of reduction in DSS.

On comparing GA scores of improvements of calcitriol and calcipotriol groups at 12 weeks, there was no statistically significant difference between the groups $(P>0.05)$ as assessed clinically (The primary efficacy criterion). The assessment of global improvement by the subject (secondary efficacy criterion) gave the same result $(\mathrm{P}>0.05)$.

Zhu et al, conducted a multicentric, randomized, investigator masked, parallel comparison of the efficacy and safety of twice daily applications of calcitriol $3 \mu \mathrm{g} / \mathrm{g}$ vs. calcipotriol $50 \mu \mathrm{g} / \mathrm{g}$ in subjects with mild to moderate chronic plaque psoriasis for 12 weeks. ${ }^{10}$ A total of 250 subjects of both genders were recruited. There was statistically significant difference in favour of calcipotriol in terms of decrease in DSS at all post baseline time-points ( $p<0.01$ ) i.e., week 2 , week 4 , week 8 and week 12. At week 12, calcitriol demonstrated to be non-inferior to calcipotriol for global improvement as assessed clinically by the investigator and also as assessed by the subject. These results counterbalance the clinical relevance of the significantly superior DSS decrease obtained with calcipotriol. Regarding efficacy of the treatment, he concluded that calcitriol $3 \mu \mathrm{g} / \mathrm{g}$ ointment administered twice daily over a 12 weeks period demonstrated similar efficacy to calcipotriol $50 \mu \mathrm{g} / \mathrm{g}$.

Present study was in concurrence with the above study in showing similar efficacy of calcitriol $3 \mu \mathrm{g} / \mathrm{g}$ ointment and calcipotriol $50 \mu \mathrm{g} / \mathrm{g}$ ointment in the treatment of chronic plaque psoriasis. But reduction in DSS and global 
assessment of improvement scores showed no significant difference between the groups in our study, whereas the above study showed significant difference in favour of calcipotriol in terms of decrease in DSS.

Ortonne et al, compared the safety and efficacy of calcitriol $3 \mu \mathrm{g} / \mathrm{g}$ ointment and calcipotriol $50 \mu \mathrm{g} / \mathrm{g}$ ointment in a multicentric, randomized, investigator blinded, left-right comparison in mild to moderate chronic plaque psoriasis affecting sensitive areas: face, hairline, retroauricular and flexural areas. ${ }^{15}$ In the 75 subjects, both treatments led to clearing of at least one target lesion in $28 \%$ of the subjects each. Global assessment of improvement from baseline by the investigators was significantly greater for the calcitrioltreated lesions. Regarding efficacy he concluded that calcitriol ointment was more effective than calcipotriol ointment in the treatment of psoriasis in sensitive areas.

In the present study, authors did not have plans within the study protocol to evaluate the efficacy of both treatments in sensitive zones. Therefore, authors could not confirm the results of the above mentioned study.

\section{Assessment of safety}

\section{Clinical assessment of cutaneous safety}

The cutaneous safety score as assessed clinically was higher with calcipotriol group than with calcitriol group (mean scores 0.28 vs 0.04 ) showing better local tolerance of calcitriol. $20 \%$ of patients developed treatment related adverse events in calcipotriol group. These were corresponding to 'mild' and 'moderate' local reactions. Only $4 \%$ of patients had 'mild 'local reactions calcitriol. But this difference was not statistically significant $(\mathrm{P}$ $>0.05)$. In both the groups, the local reaction subsided within 2-3 days without the application of any other medication and did not necessitate discontinuation of the treatment.

\section{Assessment of cutaneous discomfort by the subject}

The cutaneous discomfort score as assessed by the subject was higher with calcipotriol group than with calcitriol group (mean scores 0.36 vs 0.04 ) showing better local tolerance of calcitriol. $24 \%$ patients reported cutaneous discomfort in calcipotriol group corresponding to 'mild' and 'moderate' discomfort. Only $4 \%$ patients had a 'mild' burning sensation with calcitriol resulting in a statistically significant superiority for calcitriol $(\mathrm{P}<0.05)$.

The mentioned study conducted by Zhu et al, confirmed the better safety profile of calcitriol, with scores for cutaneous safety and cutaneous discomfort being significantly better with calcitriol than with calcipotriol (more than three times more adverse events were reported for subjects who received calcipotriol). ${ }^{10}$

In the mentioned study conducted by Ortonne et al, seven subjects experienced cutaneous adverse events with calcipotriol compared to one subject reporting one cutaneous adverse event with calcitriol. ${ }^{15}$ Calcitriol $3 \mu \mathrm{g} / \mathrm{g}$ was significantly better tolerated than calcipotriol $50 \mu \mathrm{g} / \mathrm{g}$ in terms of perilesional erythema, perilesional edema, stinging and burning. Similarly, the subjective evaluation of local tolerability and global preference were in favour of calcitriol.

In the present study, even though calcitriol $3 \mu \mathrm{g} / \mathrm{g}$ ointment showed better safety profile over calcipotriol $50 \mu \mathrm{g} / \mathrm{g}$ ointment, it was statistically not significant clinically. But it showed significant better safety profile of calcitriol $3 \mu \mathrm{g} / \mathrm{g}$ over calcipotriol $50 \mu \mathrm{g} / \mathrm{g}$ when assessed by the subject.

Thus, the results of present study show similar efficacy of calcitriol $3 \mu \mathrm{g} / \mathrm{g}$ ointment and calcipotriol $50 \mu \mathrm{g} / \mathrm{g}$ ointment in the treatment of stable chronic plaque psoriasis, whereas calcitriol has got better safety profile in terms of local tolerance and induced less treatment related adverse events.

Limitations of the study includes post treatment remission free studies was not done due to difficulty in follow up of patients. As psoriasis is a relapsing disease this would have helped us to know the remission period of the drugs used in treatment. The study did not evaluate the efficacy and safety of both the treatments in sensitive zones. The study did not consider influence of seasonal variations of disease on the effect of treatment, as most patients experience worsening of their skin lesions during winter.

\section{Funding: No funding sources}

Conflict of interest: None declared

Ethical approval: The study was approved by the Institutional Ethics Committee

\section{REFERENCES}

1. Griffiths CEM, Barker JNWN. Psoriasis. In: Burns T, Breathnach S, Cox N, Griffiths C, editors. Rook's textbook of Dermatology. vol 1. 8th ed. UK: WileyBlackwell; 2010. p. 20.1-20.60.

2. Dogra S, Yadav S. Psoriasis in India: Prevalance and pattern. Indian J Dermatol Venereol Leprol. 2010; 76(6):595-01.

3. Gudjonsson JE, Elder JT. Psoriasis. In: Goldsmith LA, Katz SI, Gilchrest BA, Paller AS, Leffell DJ, Wolff K, editors. Fitzpatrick's Dermatology in General Medicine. 8th ed. USA: McGraw Hill; 2012:197-231.

4. Richardson SK, Gelfand JM. Update on the natural history and systemic treatment of psoriasis. Advances Dermatol. 2008;24:171.

5. Hussain I, Haroon TS. Comorbidities in psoriasis and their therapeutic implications. J Pakistan Assoc Dermatol. 2016 Dec 24;19(2):63-5.

6. Singh G, Aneja SP. Cardiovascular comorbiditiy in psoriasis. Indian J Dermatol. 2011 Sep;56(5):553-6.

7. Blauvelt A. New concepts in the pathogenesis and treatment of psoriasis: key roles for IL-23, IL-17A and 
TGF- $\beta 1$. Expert Rev Dermatol. 2007 Feb 1;2(1):6978.

8. Aschoff R, Wozel G, Meurer M. Topical treatment of psoriasis: a systematic update. Dermatol J Dermatol Venereol Related Fields. 2003 Mar;54(3):237-41.

9. Sweetman SC, editor. Martindale: The complete drug reference. 37 th edition. London: Pharmaceutical press; 2011:1716-64.

10. Zhu X, Wang B, Zhao G, Gu J, Chen Z, Briantais P, Andres P. An investigator-masked comparison of the efficacy and safety of twice daily applications of calcitriol $3 \mu \mathrm{g} / \mathrm{g}$ ointment vs. calcipotriol $50 \mu \mathrm{g} / \mathrm{g}$ ointment in subjects with mild to moderate chronic plaque-type psoriasis. J Eur Acad Dermatol Venereol. 2007 Apr;21(4):466-72.

11. Langner A, Stapor W, Ambroziak M. Efficacy and tolerance of topical calcitriol $3 \mu \mathrm{g} / \mathrm{g}$ in psoriasis treatment: a review of our experience in Poland. $\mathrm{Br} \mathbf{J}$ Dermatol. 2001;144(suppl.58):11-16.

12. Lebwohl M, Menter A, Weiss J, Clark SD, Flores J, Powers J, et al. Calcitriol 3 microg/g ointment in the management of mild to moderate plaque type psoriasis: results from 2 placebo-controlled, multicenter, randomized double-blind, clinical studies. J Drugs Dermatol. 2007 Apr;6(4):428-35.

13. Ashcroft DM, Po AL, Williams HC, Griffiths CE. Systematic review of comparative efficacy and tolerability of calcipotriol in treating chronic plaque psoriasis. BMJ. 2000 Apr 8;320(7240):963-7.

14. Scott LJ, Dunn CJ, Goa KL. Calcipotriol ointment. A review of its use in the management of psoriasis. Am J Clin Dermatol. 2001;2(2):95-120.

15. Ortonne JP, Humbert P, Nicolas JF, Tsankov N, Tonev $\mathrm{SD}$, Janin A, et al. Intra-individual comparison of the cutaneous safety and efficacy of calcitriol $3 \mu \mathrm{g} g-1$ ointment and calcipotriol $50 \mu \mathrm{g} \mathrm{g}-1$ ointment on chronic plaque psoriasis localized in facial, hairline, retroauricular or flexural areas. Br J Dermatol. 2003 Feb;148(2):326-33.

Cite this article as: Hugar L, Ramesh $\mathrm{H}$. A comparative study of efficacy and safety of topical calcitriol and topical calcipotriol in stable chronic plaque type psoriasis. Int J Basic Clin Pharmacol 2019;8:402-8. 